\title{
Partial agenesis of the corpus callosum with interhemispheric lipoma: Case report
}

\author{
Abdelilah Mouhsine ${ }^{1, *}$, Ahmed Belkouch ${ }^{2}$, ElMehdi Atmane ${ }^{1}$, Redouane Rokhssi ${ }^{1}$, \\ Youssef Berrada $^{1}$, Lahcen Belyamani ${ }^{2}$, M'barek Mahfoudi $^{1}$, Abdelghani EIFikri ${ }^{1}$ \\ ${ }^{1}$ Department of Radiology Military Hospital of Avicenna, Faculty of Medicine and Pharmacy, Marrakech, Morocco \\ ${ }^{2}$ Emergency Department, Military Hospital of Instruction Mohamed V, Faculty of Medicine and Pharmacy, Rabat, Morocco
}

\section{Email address:}

abdelilahmouhsine@yahoo.fr (A. Mouhsine), belkouch1@gmail.com (A. Belkouch)

\section{To cite this article:}

Abdelilah Mouhsine, Ahmed Belkouch, ElMehdi Atmane, Redouane Rokhssi, Youssef Berrada, Lahcen Belyamani, M'barek Mahfoudi, Abdelghani ElFikri. Partial Agenesis of the Corpus Callosum with Interhemispheric Lipoma: Case Report. International Journal of Medical Imaging. Vol. 2, No. 4, 2014, pp. 92-95. doi: 10.11648/j.ijmi.20140204.12

\begin{abstract}
The agenesis of the corpus callosum and lipoma is a very rare association. We report the case of an 18 -years old young patient with no history who was admitted to the emergency department for generalized seizures and atypical headache, the onset of symptoms dated back to two months, neurological examination was normal. The brain computed tomography scan and the magnetic resonance showed the lipoma and the partial agenesis of the corpus callosum.
\end{abstract}

Keywords: Corpus Callosum, Lipoma, Agenesis, Imaging

\section{Introduction}

Corpus callosal lipoma (CCL) is a very rare benign tumor. It is often classified in the category of "obstructive" anomalies $(1,2)$. The clinical picture is very variable, it can be asymptomatic, or expressed by Seizures, or intellectual retardation. The diagnosis is based upon imagery by $\mathrm{CT}$ scan and MRI.

\section{Case Presentation}

An 18 years old young patient with no history presented to the emergency department for generalized seizures and atypical headache, the onset of symptoms dated back to two months. The clinical examination was normal, lumbar puncture was not performed.

A brain CT scan was performed and showed an inter hemispheric fat density ovoid formation, measuring about $40 \times 30 \mathrm{~cm}$ along major axis, inside that formation we noted arcuate peripheral calcifications compressing the ventricular junctions on both sides with suspected partial agenesis of the corpus callosum (figure1).

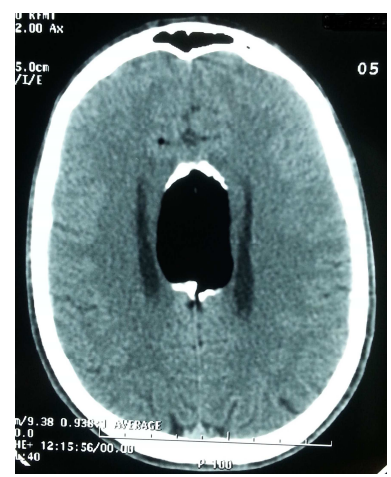

Figure 1. CT scan without contrast showing inter hemispheric fat density ovoid formation, with arcuate peripheral calcifications.

We completed the examination by MRI which confirmed the partial agenesis of the corpus callosum more specifically of the body and splenium. It revealed the inter hemispheric lesion, described above, which was in high fat signal in $\mathrm{T} 1$ and $\mathrm{T} 2$ and was not modified by injection of gadolinium. This lesion showed arcuate peripheral hyposignals formations corresponding to calcifications (FIG 2ab,3ab,4), it was also hypointense on SPT1FAT SAT. 

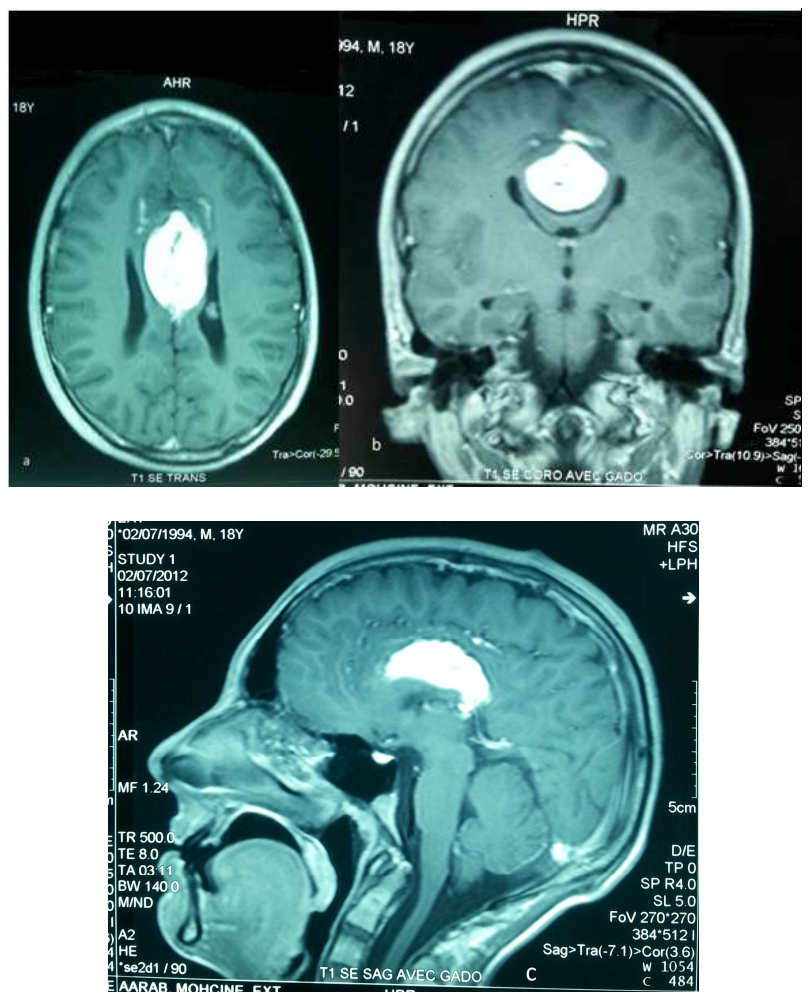

Figure 2. a-MRI on axial cut T1 revealing the interhemispheric high signal lesion, $b$ and c-MRI on coronal and sagittal cut T1 after gadolinium injection showing the same unchanged lesion.

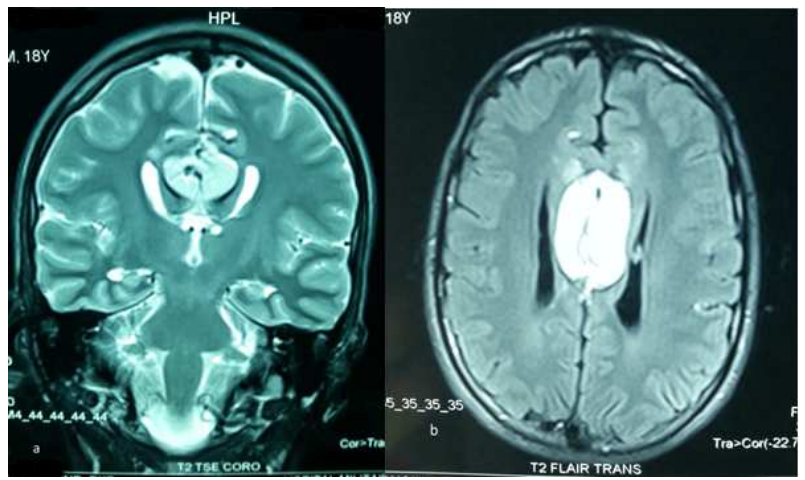

Figure 3. $a$ - MRI on coronal cut T2 revealing the interhemispheric high signal lesion, $b$ - MRI on axial cut flair revealing the interhemispheric high signal lesion

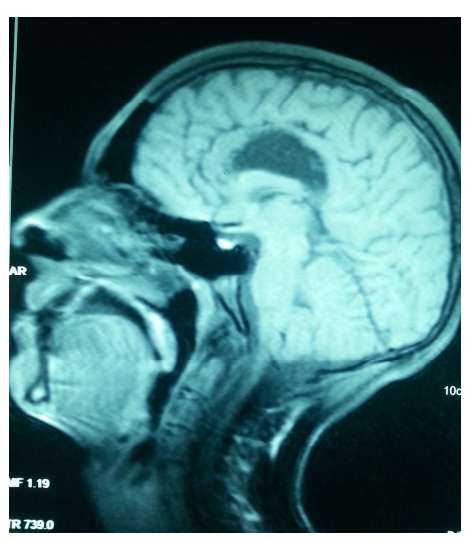

Figure 4. MRI in sagittal cut T1 fat sat showed an hyposignal lesion.
Given that the patient was stabilized under medical treatment, Surgery was avoided.

\section{Discussion}

The agenesis of the corpus callosum is usually associated with other abnormalities such as:

Interhemispheric cyst, lipoma, malformations of the cerebral cortex (heterotopia, lissencephaly) and/or an Arnold-Chiari malformation. II.

The agenesis of the corpus callosum associated with lipomas is often classified in the category of "obstructive" anomalies (2). The pathogenesis of such malformation is the premature disjunction of the neural ectoderms and skin before the closure of the neural tube. The perineural 
mesenchyme exposed to the open neural canal presents a lipomatous transformation. The compression exerted by these lipomas in the path of normal development of the corpus callosum backwards would cause frequent agenesis of this formation $(50 \%)(3,4)$. This defect depends on several factors including the size of the obstructive mass, and her rostro-caudal situation [5], [6].

Lipoma is often located on the midline or in contact and the most common abnormality associated is partial or complete commissural agenesis.

Lipomas which are not located in the path of the commissure associated with commissural agenesis suggest that there are cellular interactions between normal brain development and the development of the meninges. For example, Mehta [4] reported the case of a child with symptomatic congenital CMV infection who had a lipoma associated with complete agenesis of the corpus callosum; the link between early CMV infection and lipoma of the median line associated with agenesis of the corpus callosum is strongly suggested.

According to Raybaud, lipomas are part of the group of "complex" agenesis associating meningeal with commissural anomalies [7].

For lipomas or cysts, various studies on short series report a better prognosis in none "complex" agenesis [6], [7], [8], [9]. The clinical picture is very variable, it is asymptomatic in $10-50 \%$ of cases, it is expressed by Seizures in $50 \%$ of cases, and intellectual retardation in $20-40 \%$ [10].

The transfontanellar ultrasound shows median hyperechoic lipoma lesion and above all, it perfectly reveals the association of a total or more often a partial agenesis of the corpus callosum. It shows also, In the Coronal section, the cranial expansion of the third ventricle, the displacement from inside to outside of the frontal horns like a "bull's head" by the Probst bundles, and finally in the sagittal sections it shows the radial aspect of some convolutions.

CT scan and MRI are the gold standard [10, 11, and 12]:

The CT scan shows the lipoma with great certainty as a median mass at the knee or the anterior part of the corpus callosum, well-limited, with negative density (-50 to -100 $\mathrm{HU})$, whereas in our case the lipoma is centered on the splenium and the posterior part of the corpus callosum, with curvilinear calcification, without enhancement after contrast injection [10].

The CTscan recognizes a craniofacial dysraphism and specify the extension of some types of lipomas:

- Forward into the interhemispheric fissure, or in soft subcutaneous frontal tissue (frontal bone defect)

- Upward into the interhemispheric fissure

- Backward to the posterior part of the corpus callosum

- Downward, between the lateral ventricles or through the choroidal fissure, to the choroid plexus.

In our case MRI helped identify readily the fatty lesion hyperintense in $\mathrm{T} 1$, the islands of hypo intensity on T1-weighted sequences may correspond either to outbreaks of calcifications or to the anterior cerebral arteries included within the lipoma which are often dilated.
The differential diagnosis of this lesion is dermoid cyst, teratoma, craniopharyngioma and epidermoid tumors $[13,14,15]$.

Surgery has no place in the treatment of these lipomas; they are neither malignant nor invasive, for several reasons: the anterior cerebral arteries included within the lipoma are almost inevitably damaged during attempted resection, collagen capsule frequently adheres to adjacent brain tissue, epilepsy, the most common symptom is not always improved by surgery, and some patients can live with without engaging the prognosis [10].

\section{Conclusion}

Lipoma and agenesis of the corpus callosum is a rare association. It is usually classified in the category of obstructive anomalies. The clinical picture is very variable. CT and MRI allow positive diagnosis, assessment of the lesions and therapeutic orientation.

\section{References}

[1] Hetts SW, Sherr EH, Chao S, Gobuty S, Barkovich AJ. Anomalies of the corpus callosum: an MR analysis of the phenotypic spectrum of associated malformations. AJR Am J Roentgenol. 2006 Nov; 187(5):1343-8.

[2] Jinkins JR, Whittemore AR, Bradley WG. MR imaging of callosal and corticocallosal dysgenesis. AJNR Am J Neuroradiol 1989; 10: 339-44.

[3] M Mordefroid [1], S Grabar [2], Ch André [1], V Merzoug [1], ML Moutard [3], C Adamsbaum [1], Agénésie partielle du corps calleux de l'enfant. Journal de radiologie Vol 85, $\mathrm{N}^{\circ}$ 11 - novembre 2004 pp. 1915-1926

[4] Mehta NM, Hartnoll G. Congenital CMV with callosal lipoma and agenesis. Pediatr Neurol 2001; 24: 222-4.

[5] Griebel ML, Williams JP, Russell SS, Spence GT, Glasier $\mathrm{CM}$. Clinical and developmental findings in children with giant interhemispheric cysts and dysgenesis of the corpus callosum. Pediatr Neurol 1995;13:119-24.

[6] Doffe L, Adamsbaum C, Rolland Y, Robain O, Ponsot G, Kalifa G. Corpus callosum agenesis and parasagittal interhemispheric cyst. J Radiol 1996;77:427-30.

[7] Haverkamp F, Heep A, Woelfle J. Psychomotor development in children with early diagnosed giant interhemispheric cysts. Dev Med Child Neurol 2002;44:556-60.

[8] Sommer IE, Smit LM. Congenital supratentorial arachnoidal and giant cysts in children: a clinical study with arguments for a conservative approach. Childs Nerv Syst 1997;13:8-12.

[9] Yamasaki F, Kodama Y, Hotta T, Taniguchi E, Eguchi K, Yoshioka H. Interhemispheric arachnoid cyst in the elderly: case report

[10] DÉLRIO FAÇANHA SILVA *, MÁRCIA MARQUES LIMA **, CÉSAR O. OLIVEIRA **, WILLIAM N,OLIVEIRA, RENATO ANGHINAH, JOSE GERQLDO CAMARGO LIMA AGENESIS AND LIPOMA OF CORPUS CALLOSUM: CASE REPORT. Arq Neuropsiquiatr 1995, 53( 3.B) 
[11] Atlas SW, Zimmerman RA, Bilaniuk LT, Rorke L, Hackney DB, Goldberg HI, Grossman RI. Corpus callosum and limbic system: neuroanatomic MR evaluation of developmental anomalies. Radiology 1986, 160:355-362. 